\title{
Homicidios e investigación criminal en México
}

\section{Rodrigo Meneses Reyes, ${ }^{*}$ Miguel Quintana Navarrete**}

Perfiles Latinoamericanos, 24(48)

2016 | pp. $297-318$

DOI: $10.18504 / \mathrm{pl} 2448-012-2016$

\section{Resumen}

Este documento analiza dos tipos de homicidio: aquellos en donde las autoridades lograron determinar el motivo del homicidio, y los registrados como "homicidios sin motivo conocido". El objetivo es comprender y explicar los factores que confluyen en una investigación criminal y que contribuyen a clasificar un evento homicida como "conocido" o "desconocido". Nuestros hallazgos indican que la edad ( $<10$ ańos), el lugar (interiores) y el consumo de sustancias (drogas) son elementos clave para explicar la manera en que las investigaciones sobre homicidio son procesadas por el sistema de justicia criminal en México.

\begin{abstract}
This paper analyzes two types of homicide: those where the authorities were able to determine the reason for the murder, and those registered as "unknown homicides". The aim is to understand and explain the different factors that come together in a criminal investigation and thus contribute to classify an event as murder "known" or "unknown". Our findings indicate that age ( $<10$ years), location (indoors) and substance (drugs) are key elements to explain how investigation on homicide are processed by the criminal justice system in Mexico.
\end{abstract}

Palabras clave: homicidio, investigación criminal, violencia interpersonal, Ciudad de México, instituciones de justicia, víctimas.

Keywords: Homicide, criminal investigation, interpersonal violence, Mexico City, judicial institutions, victims.

* Doctor en Derecho. IJj-unam. Profesor Investigador Titular, División de Estudios Jurídicos del Centro de Investigación y Docencia Económicas (CIDE). Investigador Nacional Nivel I del sin.

** Estudiante del doctorado en Sociología, Harvard University. 


\section{Introducción}

$E$ de políticas públicas por determinar cuáles son los factores que afectan la manera en que un homicidio es procesado, resuelto o clasificado como "no resuelto" por las autoridades que participan en el sistema jurídico-criminal. Este interés se justifica desde dos perspectivas distintas, aunque complementarias. De un lado, porque para cualquier científico social resulta indispensable averiguar los límites y alcances de la información sobre la cual se construye el conocimiento. En caso contrario, se corre el riesgo de construir o reforzar una realidad sesgada, en donde caben diferentes interpretaciones científicas enfocadas en construir versiones propias de dinámicas que afectan a todos los miembros de una sociedad. De otro lado, como sostienen Regoeczi \& Riedel (2003: p. 155), en tanto científicos sociales nuestro entendimiento sobre la naturaleza del delito en la sociedad depende necesariamente de nuestra habilidad para recolectar información válida y confiable, que contribuya a describir tanto la frecuencia con que un delito ocurre como las características de sus participantes.

Desde donde se le vea, el análisis de la manera en que un caso de homicidio es procesado, clasificado y resuelto por las autoridades representa un tema relevante no solo para la construcción de interpretaciones científicas más fiables y cercanas a la realidad, sino también para la construcción de propuestas y alternativas tendientes a prevenir el delito y la violencia. Sin embargo, a pesar de lo prometedor que parece, esta área de estudios sobre el homicidio ha tenido un impacto limitado en América Latina. Esta falta de interés o preocupación, puede deberse a diferentes factores. Quizás el más importante es que, en la mayoría de las sociedades no occidentales, se da por sentada la carencia de sistemas de información confiables y desagregados, que nos permitan construir interpretaciones más fiables de la realidad (Schatz, 2010).

En este documento, sostenemos que es precisamente debido a estas carencias de información que resulta relevante averiguar cuáles son los factores que afectan la manera en que un homicidio es procesado, resuelto o clasificado como "no resuelto" por las autoridades que participan en el sistema jurídicocriminal. De manera más específica, este documento analiza los resultados de un análisis sobre todos los homicidios registrados en la Ciudad de México, durante el periodo 2009-2010, con el propósito de conocer los factores que median la capacidad de las autoridades criminales para determinar los motivos que dieron lugar al ejercicio de la violencia homicida. Esta decisión está fundada, principalmente, en el hecho de que la mayor parte de los estudios que giran sobre el tema han identificado que el "motivo del homicidio" (circumstance) es 
el predictor más importante de la resolución de estos casos (Alderden \& Lavery, 2007: pp. 118-119).

En otras palabras, pareciera que determinar el motivo de un homicidio representa un elemento importante no solo para resolverlo, sino también para averiguar la manera en que los casos son investigados. Como resultado, en este documento analizamos dos grupos de homicidios: 1) aquellos en donde las autoridades lograron determinar el motivo del homicidio, y 2) aquellos registrados como "homicidios sin motivo conocido". El objetivo es comprender y explicar el conjunto de factores que confluyen en una investigación criminal y que contribuyen a clasificar un evento homicida como "conocido" o "desconocido".

El resto del documento se divide en seis partes. En la primera sección, explicamos el contexto teórico e institucional en que se lleva a cabo esta investigación así como la pertinencia de la pregunta que planteamos. En la segunda, explicamos la manera en que nuestra investigación se inserta dentro de una corriente científica más amplia que ha demostrado la utilidad de las variables que aquí empleamos. En la tercera, describimos las características de los datos empleados así como los métodos de análisis que usamos en este documento. En la cuarta, presentamos los principales resultados y hallazgos identificados. En la quinta, discutimos las limitaciones de nuestras fuentes así como las precisiones y cautelas que se deben tener en cuenta al momento de interpretar los hallazgos aquí presentados. Finalmente, en la sexta sección, discutimos la relevancia de la información aquí expuesta, así como las principales conclusiones alcanzadas.

\section{Pertinencia de la investigación}

La falta de eficacia y eficiencia del sistema de justicia penal mexicano se ha documentado exhaustivamente en los últimos años (Arriaga, 2008), pese a que se ha establecido que "el nivel de delitos no denunciados o que no derivaron en averiguación previa en el país durante el 2013 fue de 93.8\% (cifra negra)" (INEGI, 2014: p. 1). Dentro de este entramado institucional, quizá son las procuradurías - tanto estatales como la federal — las que han sido más cuestionadas. Y existen buenas razones para ello. Según información del Centro de Investigación para el Desarrollo (CIDAC), únicamente se concluye el 21\% de las averiguaciones previas iniciadas en los fueros locales del país (México Evalúa, 2010: pp. 34-35). Datos de años previos sugieren incluso mayor rezago. En su comprehensivo estudio, Zepeda (2004: pp. 265-268) encontró que solamente en el $10 \%$ de los delitos denunciados el ministerio público presenta una acusación formal ante el juez. Estas cifras no dice nada de la calidad, la eficiencia, la transparencia y la honestidad con que las investigaciones son 
conducidas, aspectos que también han sido ampliamente criticados (véase, por ejemplo, Zepeda, 2003 y 2004; Naval, 2006; Magaloni, 2007; Pérez Correa, 2008; Azaola \& Ruiz, 2009), y que en su momento constituyeron una de las razones principales que motivaron una reforma profunda al sistema de justicia penal, en el año $2008 .{ }^{1}$

No obstante este diagnóstico, poco se conoce sobre los factores que influyen en la resolución de una investigación criminal. Magaloni (2007) señala que las prácticas arbitrarias que continúan plagando la actividad ministerial en México han impedido que se generen capacidades técnicas de investigación. En otras palabras, las procuradurías desarrollan su actividad bajo esquemas autoritarios heredados del pasado antidemocrático y burocrático, y no bajo estándares técnico-científicos. Cuestiones derivadas de este autoritarismo, como opacidad, corrupción y falta de supervisión judicial permitieron a las procuradurías manejar la delincuencia mediante la violación sistemática de los derechos procesales de los defendidos, y sin necesidad de profesionalizar y tecnificar la procuración de justicia. Otra explicación ha sido avanzada por Zepeda (2003: pp. 10-12), quien ha señalado que las procuradurías locales se encuentran saturadas, lo que provoca ineficiencia y un ejercicio discrecional y sin supervisión por parte del ministerio público para decidir cuáles asuntos son considerados como prioritarios. A su vez, estas prácticas también pueden generar corrupción.

Estas hipótesis han recibido corroboración empírica en el caso de la Ciudad de México a través de distintos trabajos cualitativos. Pérez Correa (2008), por un lado, y Azaola \& Ruiz (2009), por otro, han documentado las prácticas de distintos ministerios públicos y su relación con sus auxiliares en la investigación (policías y peritos, primordialmente). Ambos trabajos han podido detectar la falta de investigación sustantiva, más allá de la apertura y confección de un expediente de investigación criminal, así como rutinas abusivas y corruptas que esas autoridades despliegan en medio de un aparato burocrático que en nada favorece la investigación real de los delitos. En nuestra opinión, el impacto de estas problemáticas en la procuración de justicia está mediado por otros factores.

Por lo menos en teoría, deben existir ciertas características propias del evento delictivo que dificultan la investigación de ciertos casos, en ese contexto de carencia técnica, inercias autoritarias y saturación laboral. Más específicamente, este documento parte de la premisa de que es posible determinar otro tipo de elementos que influyen directamente en la resolución de una investigación criminal. Elementos que tienen más que ver con la confluencia de diferentes factores sociales en la estructuración de un evento homicida. Por lo tanto, el

$1 \quad$ El 18 de junio de 2008 fue publicada en el Diario Oficial de la Federación la reforma constitucional que sienta las bases para el establecimiento de un nuevo sistema de justicia penal en México. 
objetivo de este artículo es establecer los factores del evento delictivo que aumentan o disminuyen la probabilidad de que el asunto sea resuelto por la procuraduría. Pero ¿̇cuáles son los factores a los que nos referimos?

\section{Revisión de la literatura}

Aunque la literatura internacional sobre el tema sigue siendo escasa, parece existir un consenso en el sentido de que los porcentajes de solución de homicidios son generalmente mayores a los encontrados para otros delitos (Litwin, 2004: p. 327). Sin embargo, en los últimos años, este porcentaje ha disminuido en varios países (Regoeczi et al. 2008: pp. 142-143; Roberts, 2008; Xu, 2008; Keel et al. 2009: pp. 50-51; Ousey \& Lee, 2010; Maguire et al., 2010; Trussler, 2010). Gran parte del debate se ha centrado en la naturaleza de los factores que aumentan la probabilidad de que una investigación criminal no sea resuelta. Más específicamente, se ha tratado de validar o refutar factores asociados a dos modelos explicativos.

Por una parte, el modelo introducido por Black $(1976,1980)$ señala que la aplicación de la ley depende en buena medida del estatus social de la víctima, que se identifica con nociones de riqueza, respetabilidad y nivel cultural, además de factores personales como el género o la edad. La hipótesis que se desprende de este análisis es que si la víctima tiene un bajo estatus social la resolución de su caso es menos probable. Por otro lado, autores como Gottfredson \& Hindelang (1979) replicaron que todos los homicidios son investigados con la misma determinación en virtud de la gravedad de este tipo de delitos.

En cuanto a los análisis empíricos de estas posiciones, Puckett $\&$ Lundman (2003), y Litwin (2004), por ejemplo, llegaron a conclusiones similares al senalar que los factores "extralegales" o "discrecionales" mencionados por Black no impactan en la solución de los casos. También señalaron que a diferencia de lo que sucede con otros delitos o infracciones, en los homicidios los investigadores son evaluados exclusivamente mediante el número de casos resueltos, por lo que existe una fuerte presión organizacional para que todos los homicidios sean considerados como del mismo valor. No obstante, los hallazgos de ambos indican un impacto de la etnicidad en la resolución de asuntos. Puckett y Lundman encontraron menor probabilidad de solución en los homicidios cometidos en áreas predominantemente afroamericanas, y Litwin en los que tienen por víctima a personas latinas. ${ }^{2}$

2 La etnicidad es una de las variables cuyo impacto en la resolución de asuntos ha sido corroborado en numerosos estudios. Véanse, por ejemplo, a Litwin \& Xu (2007) y a Xu (2008). 
La explicación de ambos es que las comunidades con una alta proporción de minorías étnicas tienden a desconfiar de la policía, lo que complica la investigación de los homicidios porque un alto porcentaje de ellos depende de la información proporcionada por la comunidad. Esta desproporción no sugiere que los investigadores tengan un sesgo en contra de este tipo de casos, sino que inherentemente son más difíciles de resolver, por las características propias de la comunidad en que tuvieron lugar. Esta explicación también ha sido avanzada por otros académicos (Riedel \& Jarvis, 1998; Keel et al., 2009).

Por otro lado, Borg \& Parker (2001) identificaron una mayor probabilidad de resolución en las ciudades con más altos niveles de escolaridad y de estabilidad residencial. Aunque señalaron que estos resultados eran consistentes con la teoría del estatus social de Black, no descartaron otras posibles explicaciones. Litwin \& Xu (2007: p. 111) también concluyen que la teoría de Black tiene soporte empírico en su estudio, y que futuras investigaciones deben considerarla. A continuación se revisa la literatura sobre cada uno de los predictores que serán analizados en este trabajo.

\section{Armas}

Uno de los resultados más replicados es el impacto del tipo de arma utilizado. En general, aquellas armas, cuyo uso permite al agresor no tener contacto con la víctima - como una de fuego, por ejemplo-, dejan menos huellas del delito y, por lo tanto, dificultan la investigación policial. Por el contrario, las que reclaman que el agresor se acerque a la víctima para ser efectivas dejan más rastros físicos (cabello, sangre) e incrementan la probabilidad de identificación del agresor (Riedel \& Rinehart, 1996; Regoeczi et al., 2000; Puckett \& Lundman, 2003; Litwin, 2004; Alderden \& Lavery, 2007; Litwin \& Xu, 2007; Xu, 2008; Jarvis \& Regoeczi, 2009). Como lo ha señalado Litwin (2004: p. 332), pese a que las armas de fuego también proveen a los investigadores de pruebas (residuo de pólvora o información balística, por ejemplo), estas son menos importantes para identificar al agresor que para confirmar su identidad una vez que es detenido.

Estos resultados también han sido observados en otros lugares. Por ejemplo, en Trinidad y Tobago, Agozino, Bowling, Ward \& Bernard (2009: p. 292) han señalado que los homicidios cometidos con arma de fuego tienen un índice de resolución mucho menor a los cometidos por otros medios (20 vs. 62\%). De forma similar, Roberts (2008: p. 139) encontró que en Japón, aproximadamente $73 \%$ de los homicidios cometidos con arma de fuego fueron resueltos, mientras que el índice para armas de contacto fue de $93 \%$ (entre 2000 y 2004). Por su parte, $\mathrm{Xu}$ (2008) encontró un efecto similar en uno de sus modelos, 
pero desapareció una vez que incluyó los controles de series de tiempo en su análisis longitudinal.

En este documento, las distintas categorías originales de la variable de arma homicida se dicotomizaron en dos opciones: arma de fuego (0) y otra arma (1). En esta segunda se incluyen los instrumentos homicidas de contacto, como cuchillos o cualquier arma con filo, objetos romos y armas personales (manos y pies), ya sea que hayan causado la muerte por traumatismo o por asfixia. La categoría de referencia es la de arma de fuego. Esta operacionalización ha sido utilizada en otros trabajos.

\section{Edad}

Otro hallazgo consistente en la literatura ha sido que los casos con víctimas más jóvenes (niños) tienen mayor probabilidad de ser resueltos, mientras que aquellos con víctimas de la tercera edad tienen menos (Litwin, 2004; Alderden \& Lavery, 2007; Litwin \& Xu, 2007; Roberts, 2007; Regoeczi et al., 2008; Jarvis \& Regoeczi, 2009). ${ }^{3}$ Esto se debe a que los homicidios de niños son típicamente cometidos por sus padres o por sus cuidadores, pues tienen menos contacto con el exterior y en general se ven menos involucrados en actividades riesgosas (Puckett \& Lundman, 2003; Alderden \& Lavery, 2007). Esto facilita la búsqueda de los culpables y la solución de los casos. Sin embargo, en su análisis longitudinal en la ciudad de Chicago, Xu (2008) no encontró que esta variable fuera estadísticamente significativa. Siguiendo esta literatura y las operacionalizaciones empleadas en otros estudios, aquí se transformó la variable ordinal original en dicotómicas para víctimas de 10 años o menores (no=0, sí=1) y de 61 años o mayores (no=0, sí=1), siendo la categoría de víctimas entre 11 y 60 años la omitida de referencia.

\section{Género}

Uno de los hallazgos más sólidos identificados por los estudios criminológicos durante las últimas décadas, sostiene que en el estudio y cuantificación de la violencia homicida, la población del género masculino se encuentra sobrerrepresentada. Esto implica, en resumidas cuentas, que los hombres son más vulnerables a perder la vida en medio de un evento homicida que las mujeres (Miethe \& Regoeczi, 2004). Sin embargo, en el caso de las investigaciones que tratan

3 Sin embargo, otros autores no encontraron diferencias (Riedel \& Rinehart, 1996; Wellford \& Cronin, 1999). 
sobre la resolución de los homicidios, es normalmente aceptado que las víctimas del género femenino presentan menos probabilidad de que sus casos sean resueltos (Regoeczi et al., 2000; Alderden \& Lavery, 2007; Litwin \& Xu, 2007). $\mathrm{Xu}$ (2008) tampoco encontró diferencias significativas entre ambos géneros en su estudio mencionado. Esta variable se operacionalizó en dos categorías, para masculino (0) y femenino (1).

\section{Lugar}

De acuerdo con la literatura que versa sobre el tema, hay dos principales razones por las que este factor es importante para la resolución de casos. Por un lado, puede ser un indicio para localizar al culpable. Por ejemplo, un homicidio en un hogar puede sugerir que el agresor es un familiar o conocido de la víctima. De hecho, es en hogares donde previas investigaciones se ha encontrado mayor probabilidad de solución (Wellford \& Cronin, 1999; Litwin, 2004; Litwin \& $\mathrm{Xu}, 2007) .{ }^{4}$ Por otro lado, los eventos homicidas en espacios públicos son más propicios para la existencia de testigos, lo que en otros estudios también ha resultado significativo (Litwin \& Xu, 2007; Xu, 2008).

La variable original de lugar de fallecimiento incluía muchas categorías que en atención a las investigaciones previas, principalmente en Jarvis \& Regoeczi (2009), se agruparon en tres: 1) hogar/espacio residencial, 2) otros lugares interiores (como hospitales, consultorios, terminales aéreas o de autobús, restaurantes, escuelas y universidades, prisiones y otros centros de reclusión, edificios comerciales y de oficinas, bares y antros, iglesias, entre otros similares), y 3 ) lugares exteriores (como calles y avenidas, parques, lotes baldíos, campos y páramos, estacionamientos, lugares en construcción, canales, entre otros). Posteriormente, se construyeron variables dicotómicas para hogar/espacio residencial (no= 0 , sí=1) y para otro lugares internos (no=0, sí=1), de las que se excluyó la categoría de lugares externos.

\section{Drogas}

Este factor ha sido poco estudiado en el contexto de la resolución de asuntos. Entre los artículos revisados, solo uno tomó en consideración el consumo de drogas como predictor de los casos resueltos (Wellford \& Cronin, 1999). En ese documento, Wellford y Cronin reportaron que algunos fenómenos relacionados con

4 Aunque $\mathrm{Xu}$ (2008) no encontró una diferencia significativa con respecto a homicidios en otro tipo de lugares. 
el consumo y la venta de drogas dificultan la resolución de los casos. Por ejemplo, entre los asuntos estudiados los que contaban con víctimas que tenían un historial de uso de drogas tuvieron $46 \%$ menos probabilidad de ser resueltos, $40 \%$ menos probabilidad si el historial de la víctima incluía relaciones sociales con narcotraficantes y usuarios de drogas, y $35 \%$ menos probabilidad si la víctima tenía un arresto previo por alguna cuestión relacionada con drogas.

En cuanto a la interpretación de este fenómeno, Litwin (2004: p. 333) menciona que en los homicidios relacionados con drogas o pandillas es posible que los testigos sean más renuentes a declarar por temor a represalias. No obstante, los autores no conocemos estudios en los que se haya analizado el impacto del consumo de drogas por parte de la víctima de homicidio en el nivel de investigación de los casos correspondientes. Este factor se analizó a través de la creación de una variable dicotómica para determinar si la víctima había ingerido alguna droga previamente a su muerte (no=0, sí=1).

\section{Alcohol}

Los autores de este documento no conocemos de algún trabajo académico sobre los índices de resolución de homicidios que haya contemplado el consumo de alcohol como probable predictor. Sin embargo, investigaciones en otros países han establecido que el alcohol suele tener un rol determinante en muchos eventos homicidas (ops, 2010). En México, la poca información disponible también lo sugiere (Meneses \& Quintana, 2013). Al igual que en el caso de la variable de uso de drogas, este factor se dicotomizó para conocer si la víctima había consumido alcohol antes de su fallecimiento (no=0, sí=1).

\section{Datos y métodos}

Para el análisis de datos, se utilizó la técnica de regresión logística binaria con el fin de determinar cuáles de las variables independientes predicen significativamente el nivel de investigación después de controlar el impacto del resto de las variables en la ecuación. Esta selección se debe a que la variable dependiente es dicotómica (se ignora el motivo $=0$, se conoce el motivo=1). En el análisis se introdujeron todos los predictores simultáneamente. Antes de pasar a la presentación de los resultados, sin embargo, conviene aclarar algunas particularidades de la información aquí recopilada.

En México no existe un sistema de información sobre justicia penal, sino que existen distintas fuentes de información que no son compatibles entre sí 
(Ramírez, 2012). Más aún, las distintas instituciones generalmente presentan datos agregados por variable, no por caso, lo que imposibilita darle continuidad a un asunto a través de las distintas etapas del procedimiento penal. ${ }^{5}$ Para soslayar estos problemas, en el presente estudio se utiliza la base de datos de muertes violentas del Servicio Médico Forense del Distrito Federal, dependiente del Tribunal Superior de Justicia.

En términos forenses, el concepto de "muertes violentas" se equipara con el de "homicidios dolosos", que es definido por la mayoría de los códigos penales de México como la acción u omisión de una persona que priva de la vida a otra con esa intención. Se trabajó sobre los 2204 homicidios registrados entre los años 2009 y 2010. Este periodo se seleccionó en virtud de que es el único para el que se cuenta con información caso por caso. Las variables incluidas en la base de datos y que se considerarán en este estudio son: edad de la víctima al morir, género de la víctima, lugar de muerte, tipo de arma utilizada y el resultado del examen toxicológico (presencia de alcohol y/o drogas).

En Estados Unidos, el Federal Bureau of Investigation define al índice de solución de casos como la razón entre el número de casos resueltos y el número de casos reportados a la policía en un periodo determinado (Borg \& Parker, 2001: p. 439). Un caso se puede resolver de dos formas distintas. La primera —y más común - requiere el arresto del sospechoso, el levantamiento de cargos formales y la remisión del caso a un juzgado o tribunal. La segunda se refiere a los asuntos "resueltos excepcionalmente", que son aquellos considerados como solucionados pero sin que se haya hecho arresto alguno, debido a una circunstancia externa que lo impida. Ejemplos de esta forma de resolución son la muerte del ofensor o la negativa de la víctima a colaborar con la procuración una vez que el ofensor ha sido identificado (Riedel \& Boulahanis, 2007: pp. 152-153; Jarvis \& Regoeczi, 2009: pp. 174-175).

En México, en términos generales, una vez iniciada la investigación criminal el Ministerio Público puede tomar dos tipos de determinaciones: ejercer o no ejercer la acción penal, lo que se le conoce como hacer llegar o no la investigación criminal ante un juez (Código Federal de Procedimientos Penales,

5 Típicamente, en México, el proceso penal inicia con una "denuncia" del hecho delictivo ante el fiscal, y termina con una "sentencia" judicial. Sin embargo, en medio de estas decisiones existen diversas posibilidades. Por ejemplo, al tener noticia del evento delictivo, el fiscal puede iniciar o no una investigación criminal —lo que normalmente depende de qué tan verosímil resulta el caso-. Asimismo, una vez iniciada la investigación criminal, el fiscal debe allegarse de diferentes elementos para sustentar su acusación. En caso de que estos elementos sean reunidos, el fiscal envía el expediente de investigación al juez encargado de decidir sobre el caso. Sin embargo, el fiscal también puede decidir que la investigación no ha reunido los elementos suficientes para ser conocida por un juez, y entonces archivar el caso o simplemente declararlo como no acreditado. 
artículos 136 y 137). Como se puede apreciar, no existe una correspondencia perfecta entre los supuestos de resolución de casos en los que se ha basado la mayoría de la literatura aquí revisada, y los que existen en México.

Por ejemplo, en Estados Unidos, los detectives policiales resuelven un caso cuando identifican al sospechoso y lo detienen; en México, el ministerio público "resuelve" un caso al enviar el expediente de la investigación criminal a un juez, sin necesidad de la detención del sospechoso. ${ }^{6}$ Pese a las diferencias, en uno y otro se habla de la etapa de investigación previa al proceso penal seguido ante la autoridad judicial, por lo que en el caso de México sería posible utilizar la averiguación previa y la resolución del Ministerio Público que da fin a esa etapa para referirnos al índice de resolución de casos.

Sin embargo, la falta de información sistemática sobre las investigaciones criminales conocidas por un juez en México conlleva a la necesidad de buscar una medida que permita acercarnos al grado de investigación de un caso criminal. En tal situación, consideramos que la capacidad del ministerio público para determinar los motivos del homicidio representa una buena manera de aproximarnos al grado de investigación criminal. Se parte de la premisa de que un asunto en que se ha determinado el motivo, permite que el fiscal o ministerio público envíe el expediente completo ante la autoridad judicial, mientras que lo contrario ocurre cuando no hay un nivel satisfactorio de investigación. ${ }^{7}$

Esta determinación se basa en que la mayor parte de la literatura que existe sobre el tema indica que solo un porcentaje muy pequeño de los casos son resueltos sin conocer el motivo del homicidio. Por ejemplo, en Estados Unidos, solo aproximadamente el 15\% de los casos son resueltos sin conocer el motivo del homicidio (Alderden \& Lavery, 2007: p. 121). De hecho, el incremento sustancial en los últimos años en el porcentaje de casos con circunstancias y motivos desconocidos en Estados Unidos se ha interpretado como una explicación del descenso en los índices de resolución (Litwin \& Xu, 2007: p. 111). En otras palabras, conocer el motivo del homicidio es uno de los factores más influyentes en la resolución de los casos (Alderden \& Lavery, 2007).

6 En realidad, es inapropiado hablar de la solución de un caso con la consignación, pues el Ministerio Público solo debe probar la probable responsabilidad del indiciado en esa etapa procesal. Pero aquí es apropiado obviar esta distinción porque de hecho la actividad "dura" de investigación del Ministerio Público termina con la consignación del caso.

7 En general, las investigaciones sobre casos de homicidio se construyen sobre tres fuentes primarias: 1) entrevistas con testigos del evento homicida o con hechos relacionados al mismo; 2) el análisis de la escena del crimen, 3) los antecedentes de la víctima, así como las opiniones de sus familiares o amigos relacionadas con las causas que pudieron suscitar el hecho (enemistades, venganzas, deudas). En cualquier caso, estos tres elementos de información pueden ser utilizados de manera independiente o complementaria en orden de establecer una hipótesis final del caso. 


\section{Resultados}

En la tabla 1 se muestran los descriptivos tanto de la variable dependiente como de las variables independientes. Como puede observarse, en seis de cada diez homicidios registrados en la Ciudad de México la autoridad ignora el motivo $(58.8 \%)$. Adicionalmente, un dato que contrasta es el poco porcentaje de víctimas que ingirieron alguna droga $(10.5 \%)$ y el alto porcentaje que bebieron alcohol antes de fallecer (59.6\%). Estos resultados confirman un hallazgo identificado por investigaciones previas que indican que, al menos en la Ciudad de México, el alcohol tiene un rol muy importante en la configuración de los eventos homicidas (Meneses \& Quintana, 2013). También destaca el alto porcentaje de homicidios que se cometen con arma de fuego $(59.4 \%) \mathrm{y}$ la sobrerrepresentación del género masculino en las víctimas de homicidio $(87.2 \%)$.

Tabla 1. Variables y descriptivos

\begin{tabular}{|c|c|c|}
\hline Variables & N & $\%$ \\
\hline \multicolumn{3}{|l|}{ Motivo } \\
\hline Conocido & 906 & 41.2 \\
\hline Desconocido & 1291 & 58.8 \\
\hline \multicolumn{3}{|l|}{ Género } \\
\hline Femenino & 280 & 12.7 \\
\hline Masculino & 1916 & 87.2 \\
\hline \multicolumn{3}{|l|}{ Edad } \\
\hline$\leq 10$ años & 52 & 02.4 \\
\hline entre 11 y 60 años & 2030 & 92.5 \\
\hline$\geq 61$ años & 114 & 05.2 \\
\hline \multicolumn{3}{|l|}{ Drogas } \\
\hline Sí consumió & 230 & 10.5 \\
\hline No consumió & 1967 & 89.5 \\
\hline \multicolumn{3}{|l|}{ Alcohol } \\
\hline Sí consumió & 1310 & 59.6 \\
\hline No consumió & 887 & 40.4 \\
\hline \multicolumn{3}{|l|}{ Lugar } \\
\hline Hogar/espacio residencial & 217 & 09.9 \\
\hline Otros interiores & 928 & 42.2 \\
\hline Exteriores & 1042 & 47.4 \\
\hline Otros & 10 & 0.50 \\
\hline \multicolumn{3}{|l|}{ Arma } \\
\hline Arma de fuego & 1304 & 59.4 \\
\hline Arma de contacto & 882 & 40.1 \\
\hline Otros & 11 & 0.50 \\
\hline
\end{tabular}


En la tabla 2, se presentan las variables independientes consideradas en el modelo y los datos relevantes para determinar su significancia estadística. Por lo demás, el modelo de regresión logística binaria fue significativo $(\mathrm{X} 2=113.888$, $8 \mathrm{gl}, p<.001)$, y tuvo un valor de pseudo R2 de .068 .

Tabla 2. Resultados de la regresión

\begin{tabular}{lcccc}
\hline Variables & $B$ & Wald & Sig. & Razón de probabilidades \\
\hline Género & 0.189 & 1.828 & .176 & 1.208 \\
Edad & & & & \\
$\quad \leq 10$ años & 2.506 & 27.508 & $.000^{*}$ & 12.259 \\
$\quad \geq 61$ años & 0.086 & 0.182 & .669 & 1.089 \\
Drogas & -0.657 & 17.125 & $.000^{*}$ & 0.518 \\
Alcohol & 0.154 & 2.817 & .093 & 1.166 \\
Lugar & & & & \\
$\quad$ Hogar/espacio residencial & 0.741 & 21.084 & $.000^{*}$ & 2.098 \\
$\quad$ Otros interiores & 0.359 & 14.382 & $.000^{*}$ & 1.432 \\
Arma & -0.086 & 0.825 & .364 & 0.918 \\
\hline
\end{tabular}

$\left.{ }^{*}\right)$ Significativos $p \leq 0.001$

Fuente: Elaboración propia a partir de SEMEFO-DF, 2009-2010

El modelo reveló algunos predictores significativos al $p \leq 0.001$ : consumo de drogas, el lugar del homicidio y una de las variables dicotómicas relativas a la edad. El factor más importante es este último. En resumidas cuentas, pareciera que en un homicidio con una víctima de 10 años o menor es $1126 \%$ más probable que las autoridades logren determinar el motivo que en un evento homicida con una víctima de entre 11 y 60 años de edad, manteniendo constantes las demás variables.

Este resultado parece reforzar que la edad temprana es un factor determinante tanto en la investigación como en la resolución de los homicidios (Litwin, 2004; Alderden \& Lavery, 2007; Litwin \& Xu, 2007; Roberts, 2007; Regoeczi et al., 2008; Jarvis \& Regoeczi, 2009), aunque en el caso de la Ciudad de México su efecto es mucho mayor. Los homicidios en los que están involucradas víctimas de la tercera edad (>60 años), por el contrario, no fueron significativamente diferentes a los de la categoría intermedia.

Por la forma en que se codificó la variable de drogas el resultado de la regresión señala que en un homicidio con una víctima que hubiere consumido droga antes de su muerte es $48 \%$ menos probable que se conozca el motivo, comparándolo con uno en el que la víctima no hubiera utilizado drogas y manteniendo constantes el resto de las variables del modelo. Hay, al menos, dos posibles interpretaciones para este resultado. La primera es que las circunstancias 
que típicamente rodean un homicidio con una víctima que ha ingerido droga dificulten la investigación. ${ }^{8}$ La segunda explicación está relacionada con el argumento de Black $(1976,1980)$, y señalaría la existencia de un patrón de discriminación hacia los consumidores de drogas, sea por el bajo estatus social que ostentan como consecuencia de ese consumo o porque se trata de sujetos involucrados en actividades ilícitas.

Esta misma explicación sugeriría, como señala un estudioso del sistema penal de la Ciudad de México, que quizá detrás de estas cifras lo que pasa es que "a los Ministerios Públicos y policías de investigación no les interesa demasiado investigar crímenes donde las víctimas eran consumidores de estupefacientes" (Fondevila, 2013). En todo caso, lo importante a notar en este documento es que el consumo de drogas, por parte de la víctima, parece representar un elemento distintivo de las averiguaciones por homicidio que se dan en la Ciudad de México, y que ha sido poco explorado por otros investigadores, tanto en México como en otras sociedades.

En cuanto al lugar, los casos en que se encontró a la víctima en el hogar o en un espacio residencial tuvieron un $110 \%$ más de probabilidades de que se identificara el motivo del homicidio, con respecto a los asuntos cuyas víctimas fueron encontradas en lugares exteriores, y con el resto de las variables de la ecuación constantes. En otros lugares interiores también fue más probable que se determinara el motivo del homicidio con respecto a esta última categoría, en $43 \%$.

Estos resultados corroboran que los homicidios en hogares se prestan con mayor probabilidad a una solución, hallazgo identificado en estudios previos (Wellford \& Cronin, 1999; Litwin, 2004; Litwin \& Xu, 2007). No obstante, algunos autores también han señalado que los espacios abiertos o exteriores son más propicios para que haya testigos de los hechos, lo que facilitaría la investigación (Litwin \& Xu, 2007; Xu, 2008).

Nuestros datos indican que, en la Ciudad de México, es más bien en espacios interiores en donde existe mayor probabilidad de que las autoridades determinen el motivo del homicidio. Esta diferencia se puede deber a bastantes factores. Uno es que las operacionalizaciones varían de un trabajo a otro, lo que sin lugar a dudas repercute en los resultados. Además, hay muchos lugares exteriores que por su lejanía o su difícil acceso minimizan la posibilidad de que existan testigos, como los páramos o los canales.

8 Por ejemplo, los testigos podrían presentar cierta reticencia a declarar en este tipo de delitos (Litwin, 2004). En el mismo sentido, podría pensarse que esta situación es el resultado de las carencias técnicas que experimentan algunas instituciones para procesar cierto tipo de pruebas forenses. 
Contrariamente, muchos lugares cerrados que son punto de reunión de mucha gente facilitan que las personas observen los hechos, como los hospitales o los centros comerciales, además de que también pueden estar provistos de tecnología de vigilancia que incrementaría las posibilidades de resolver el asunto. Otra cuestión es que los homicidios en los que las víctimas son transportadas y abandonadas en un lugar distinto al del evento homicida son por lo general más difíciles de resolver. Como lo señala un detective de la ciudad de Chicago, "la persona que abandona un cuerpo después del asesinato en otro lugar es una persona muy difícil de rastrear" (Litwin \& Xu, 2007: p. 100). Y por la naturaleza misma de este tipo de acción, esas víctimas son abandonadas en lugares exteriores, muchas veces lejanos o desolados. ${ }^{9}$

\section{Limitaciones de la investigación}

Pese a que la base de datos aquí analizada es la fuente más completa que actualmente existe en México — al menos respecto a los homicidios_-, presenta varias limitaciones que impiden la elaboración de un estudio que contraste nuestros hallazgos con los obtenidos por otros investigadores. En primer término, no contempla muchas de las variables que han sido consideradas en otros trabajos, principalmente en Estados Unidos. Entre ellas destacan el volumen y la dinámica de manejo de los casos, los recursos a disposición de los investigadores, y la experiencia de estos últimos (Borg \& Parker, 2001; Puckett \& Lundman, 2003; Litwin \& Xu, 2007; Keel et al., 2009); la influencia política, principalmente de los medios de comunicación y de la alta burocracia (Davies, 2007; Keel et al., 2009); la calidad del proceso de investigación, determinada en buena medida por el grado de acceso y uso de tecnología y de bases de datos (Wellford \& Cronin, 1999; Keel et al., 2009); el entrenamiento y profesionalización de los investigadores (Keel et al., 2009); el tiempo de respuesta, o el que pasa entre el evento homicida y la investigación (Wellford \& Cronin, 1999; Regoeczi et al., 2008); la ideología o la forma de pensar de los investigadores con respecto a ciertos tópicos (Reasons et al., 2010); si la víctima tenía antecedentes penales (Wellford \& Cronin, 1999; Litwin, 2004; Alderden \& Lavery, 2007; Litwin \& Xu, 2007); la relación entre la víctima y el ofensor (Riedel \& Jarvis, 1998; Alderden \& Lavery, 2007; Xu, 2008), y el momento

9 La actividad del narcotráfico también podría contribuir a que este tipo de homicidios sean más comunes en México que en otros países. Además, un homicidio relacionado con el narco reduce la posibilidad de que haya testigos, por temor a represalias, como sugirió Litwin (2004) en relación con los homicidios de pandillas. 
del día en el que se cometió el homicidio (Regoeczi et al., 2008; Alderden \& Lavery, 2007).

En segundo lugar, la falta de especificidad de la base de datos con respecto a la jurisdicción en donde el homicidio tuvo lugar, tampoco permite aproximarnos a aspectos estructurales del área de la Ciudad de México en la que ocurrió el evento, los cuales podrían ser vitales para validar o refutar los diversos componentes de la teoría de Black. Estos aspectos se han medido a través de distintas operacionalizaciones, como densidad poblacional, índice de homicidios y otros delitos, composición étnica, ingreso y empleo, movilidad residencial, inequidad social y económica, hogares monoparentales, educación y acceso a bienes y servicios públicos, entre otras (Borg \& Parker, 2001; Puckett \& Lundman, 2003; Litwin, 2004; Davies, 2007; Litwin \& Xu, 2007; Xu, 2008; Keel et al., 2009). La intención con estas variables es conocer el contexto social inmediato en el que ocurrió el homicidio y en el que presumiblemente se desarrollaba la víctima para determinar si influye en la resolución de los casos. Los estudios que han introducido estas variables toman como unidad de análisis alguna construcción socioespacial (barrios, ciudades) (Borg \& Parker, 2001; Davies, 2007) o han introducido ese tipo de mediciones en análisis con múltiples niveles (Puckett \& Lundman, 2003; Litwin, 2004; Litwin \& Xu, 2007; Xu, 2008).

Finalmente, no es posible determinar — a partir de la base de datos o de cualquier otra información disponible - si la investigación criminal abierta para cada homicidio fue conocida por un juez o no. Evidentemente, estas condiciones limitan el alcance del presente estudio y deja huecos que futuros trabajos deberán considerar y tratar de solucionar. Aquí se pretende iniciar la producción de conocimiento sobre este tema en México con la finalidad de eventualmente entender a fondo el fenómeno de la resolución de casos. Esto último requerirá de un estado más avanzado de la cantidad y calidad tanto de la generación como de la disponibilidad de datos.

\section{Discusión}

En este documento intentamos responder una pregunta concreta: ¿qué factores afectan la manera en que un homicidio es procesado, resuelto o clasificado como "no resuelto" por las autoridades que participan en el sistema jurídico-criminal en la Ciudad de México? Con el actual nivel de información y de conocimiento desarrollado sobre el tema en México es posible que algunos de los hallazgos aquí identificados requieran de análisis más detallados y comprensivos. Sin embargo, nuestros hallazgos parecen coincidir con algunos de los resultados 
de otros autores, lo que bien puede abrir la puerta para identificar prácticas institucionales similares en contextos y situaciones disímiles.

En este sentido, nuestros resultados indican que, al menos en la Ciudad de México, la edad ( $\leq 10$ años), el lugar (interiores) y el consumo de sustancias (drogas) son elementos clave para explicar la manera en que los casos son procesados por el sistema de justicia criminal. Las variables de edad y lugar —más específicamente la categoría de hogar/espacio residencial—, por ejemplo, pueden estar asociadas a un mismo mecanismo causal. En ambos casos es más probable que los allegados a la víctima conozcan al agresor, haciendo la resolución más sencilla. A su vez, esto también podría indicar que estos casos son aquellos que se podrían resolver de manera más inmediata, bajo las figuras de flagrancia o flagrancia equiparada.

Este detalle parece crucial para el caso de México pues, como desde hace algunos años se ha demostrado (Zepeda, 2004), uno de los rasgos distintivos de la procuración de justicia mexicana es que la gran mayoría de las investigaciones criminales que se resuelven están precedidas por una detención en flagrancia o flagrancia equiparada, un dato que se ha corroborado en estudios posteriores. ${ }^{10}$ Esto sugiere que en estos casos no hubo necesidad de llevar a cabo una investigación exhaustiva para dar por concluida la averiguación, y que la resolución será favorable al ministerio público. Siguiendo esta línea, en Estados Unidos, Alderden \& Lavery (2007) encontraron que cuando se excluían de su muestra los casos conocidos como quick clearances, y que pueden equipararse a los delitos resueltos por flagrancia en virtud del poco tiempo y nivel de investigación que requieren, la edad dejaba de tener significancia estadística. Nuestra base de datos no nos permite controlar por casos que fueron resueltos en flagrancia o flagrancia equiparada. Sin embargo, existe una fuerte presunción de que se conoce su motivo, por lo que no estarían distribuidos aleatoriamente entre los resueltos y no resueltos. En otras palabras, los casos que involucran niños menores de 10 años y con víctimas en el hogar podrían encontrarse mayoritariamente entre los que se resuelven bajo estas figuras, rápidamente y sin necesidad de investigación.

Alderden \& Lavery (2007) también señalaron que los homicidios caracterizados como expresivos tienen una mayor probabilidad de ser resueltos que los instrumentales. Aunque sin hacer específicamente esta distinción, un hallazgo ampliamente reconocido en la literatura indica que los homicidios que se cometieron en medio de otro delito (típico escenario de los homicidios instrumentales) son más difíciles de resolver (Riedel \& Rinehart, 1996; Wellford \& Cronin, 1999; Regoeczi et al., 2000; Litwin, 2004; Litwin \& Xu, 2007; Xu,

10 Véase CIDE-Encuesta a Población en Reclusión (2002, 2005, 2009).

R. Meneses Reyes, M. Quintana Navarrete | Homicidios e investigación criminal en México Perfiles Latinoamericanos, 24 (48) | FLACso México | pp. 297-318 | DoI: 10.18504/pl2448-012-2016 
2008). Los casos que involucran niños y cuya víctima se encuentra en el hogar tienen mayor probabilidad de ser expresivos, lo que también ayudaría a su más alto índice de resolución. Esta hipótesis parece reforzarse si consideramos que, de las mujeres sentenciadas por homicidio doloso en la Ciudad de México, el $50 \%$ fueron acusadas de privar de la vida a una víctima de 10 años o menor, de los cuales el 63\% eran familiares; presumiblemente un hijo menor de edad que fue privado de la vida como consecuencia de un problema de violencia intrafamiliar (CIDE-Encuesta a Población en Reclusión, 2002, 2005, 2009). ${ }^{11}$

Por lo demás, en la Ciudad de México hay una mayor proporción de homicidios instrumentales que en Estados Unidos. Investigaciones posteriores deben explorar la posibilidad de que esta circunstancia explique en buena parte los índices menores de resolución en México. También se debe destacar que las variables de género y arma no fueron significativas en esta especificación del modelo, contrario a lo que han encontrado otros trabajos. Es decir, no hay diferencias entre los asuntos con víctimas mujeres y con víctimas hombres, como tampoco entre homicidios con arma de fuego y con arma de contacto.

Dadas las limitaciones de la información disponible, no es posible saber si las características de los casos aquí analizados han variado con el tiempo. Tampoco es posible determinar si la capacidad institucional de procesamiento y resolución de homicidios ha variado, como sugieren diferentes estudios desarrollados en otros países (Regoeczi et al. 2008: pp. 142-143; Roberts, 2008; Xu, 2008; Keel et al., 2009: pp. 50-51; Ousey \& Lee, 2010; Maguire et al., 2010; Trussler, 2010). Sin embargo, a partir de 2008, las instituciones jurídicas mexicanas han intentado reformar sus prácticas, con el objetivo de mejorar las capacidades institucionales para procesar y resolver los delitos, particularmente aquellos que tienen que ver con el despliegue de la violencia homicida.

Desafortunadamente, esta reforma ha tenido lugar en un clima de violencia extrema, en donde la tasa anual de homicidios por cien mil habitantes ha pasado de 11.02 en 2000 a 22.93 en 2010, en todo el país (INEGI, 2011). Dicha tasa decreció a 21.5 para el 2012, pero continúa por arriba del promedio del continente americano (16.3), el más violento del mundo, aunque por debajo de la tasa de países como Honduras (90.4), Venezuela (53.7), Colombia (30.8) y Brasil (25.2) (UNODC, 2013: pp. 22-24). Esto no solo ha impedido la consolidación de la reforma mencionada, sino que también ha oscurecido las capacidades analíticas para determinar la manera en que se ejerce la violencia homicida cotidianamente. Básicamente, el principal problema analítico que ha traído consigo esta alza en

11 Resulta interesante advertir que, cuando se trata de la población masculina, el porcentaje de sujetos sentenciados por la comisión de un homicidio doloso en donde la edad de la víctima era de 10 ańos o menor, es de $2 \%$. La diferencia entre hombres y mujeres resulta estadísticamente significativa al $p<0.001$. 
el número de homicidios ha sido la creación de nuevas bases de datos que han pretendido disociar la violencia ejercida por el crimen organizado del resto de los homicidios que tienen lugar en el país. Sin embargo, aunque en principio parece adecuado distinguir los tipos de violencia homicida, este ejercicio ha redundado en una fragmentación cada vez mayor de la información, lo que complica el análisis y comprensión del fenómeno. En este sentido, consideramos que el análisis aquí presentado contribuye a revertir esta tendencia y, sobre todo, permite reconocer que la información ya existente puede arrojar luz tanto de los elementos que confluyen en el delito de homicidio, como de la forma en que estas investigaciones criminales son procesadas.

\section{Bibliografía}

Agozino, B., Bowling, B., Ward, E. \& Bernard, G. (2009). Guns, Crime and Social Order in the West Indies. Criminology and Criminal Justice, 9, 287-308.

Alderden, M. \& Lavery, T. (2007). Predicting Homicide Clearance in Chicago: Investigating Disparities in Predictors Across Different Types of Homicide. Homicide Studies, 11, $115-132$.

Arriaga, L. (2008). Sistema de Procuración de Justicia y Derechos Humanos en México. El Cotidiano, 23, 83-88.

Azaola, E. \& Ruiz, M. (2009). Investigadores de papel: Poder y derechos humanos entre la Policía Judicial de México. Fontamara: México.

Black, D. (1976). The Behavior of Law. Academic Press: Nueva York.

Black, D. (1980). The Manners and Customs of the Police. Academic Press: Nueva York.

Borg, M. \& Parker, K. (2001). Mobilizing Law in Urban Areas: The Social Structure of Homicide Clearance Rates. Law and Society Review, 35, 435-466.

Centro de Investigación y Docencia Económicas (CIDE). (2002, 2005, 2009). Encuesta a Población en Reclusión en el Distrito Federal y el Estado de México. Recuperado de http://biiacs -dspace.cide.edu/handle/10089/16345

Davies, H. (2007). Understanding Variations in Murder Clearance Rates: The Influence of the Political Environment. Homicide Studies, 11, 133-150.

Fondevila, G. (2013, 15 de enero). El negocio de los adictos. Reforma. 
Gottfredson, M. \& Hindelang, M. (1979). A Study of the Behavior of Law. American Sociological Review, 44, 3-18.

Ingram, M., Rodríguez, O. \& Shirk, D. (2011). Assessing Mexico's Judicial Reform: View of Judges, Prosecutors, and Public Defenders (Special report). Trans-Border Institute Joan B. Kroc School of Peace Studies. University of San Diego, San Diego.

Instituto Ciudadano de Estudios sobre la Inseguridad, A.C. (ICESI) (2009). Sexta Encuesta Nacional sobre Inseguridad, ENSI-6: Resultados Primera Parte. Recuperado de http://tinyurl .com/a5khjlf

INEGI (2014). Encuesta nacional de victimización y percepción sobre seguridad pública. Recuperado de http://www.inegi.org.mx/inegi/contenidos/espanol/prensa/Boletines/Boletin/ Comunicados/Especiales/2014/septiembre/comunica11.pdf

INEGI (2012). Encuesta nacional de victimización y percepción sobre seguridad pública.

Jarvis, J. \& Regoeczi, W. (2009). Homicide Clearances: An Analysis of Arrest Versus Exceptional Outcomes. Homicide Studies, 13, 174-188.

Keel, T., Jarvis, J. \& Muirhead, Y. (2009). An Exploratory Analysis of Factors Affecting Homicide Investigations: Examining the Dynamics of Murder Clearance Rates. Homicide Studies, 13, 50-68.

Litwin, K. \& Xu, Y. (2007). The Dynamic Nature of Homicide Clearances: A Multilevel Model Comparison of Three Time Periods. Homicide Studies, 11, 94-114.

Litwin, K. (2004). A Multilevel Multivariate Analysis of Factors Affecting Homicide Clearances. Journal of Research in Crime and Delinquency, 41, 327-351.

Magaloni, A. (2007). Arbitrariedad e Ineficiencia de la Procuración de Justicia: Dos Caras de la misma Moneda. Documentos de Trabajo, DTEJ, 26. México: CIDE.

Maguire, E., King, W., Johnson, D. \& Katz, C. (2010). Why Homicide Clearance Rates Decrease: Evidence from the Caribbean. Policing and Society: An International Journal of Research and Policy, 20, 373-400.

México Evalúa (2010). SIIS: Sistema de Índices e Indicadores en Seguridad Pública. Recuperado de www.mexicoevalua.org/wp-content/uploads/2013/03/SIIS-2010.pdf

Naval, C. (2006). Irregularidades, abusos de poder y maltratos en el Distrito Federal: La relación de los agentes policiales y del ministerio público con la población. México: FUNDAR. 
Ousey, G. \& Lee, M. (2010). To Know the Unknown: The Decline in Homicide Clearance Rates, 1980-2000. Criminal Justice Review, 35, 141-158.

Pérez Correa, C. (2008). Front Desk Justice: Inside and Outside Criminal Procedure in Mexico City. Mexican Law Review, 1, 3-31.

Puckett, J. \& Lundman, R. (2003). Factors Affecting Homicide Clearances: Multivariate Analysis of a More Complete Conceptual Framework. Journal of Research in Crime and Delinquency, 40, 171-193.

Ramírez, K. (2012). Pautas para el análisis de los delitos violentos a partir de la información estadística en México. En Echarri, C. (Ed.). Panorama estadístico de la violencia en México. México: Centro de Investigación y Estudios en Seguridad (CIEs).

Reasons, C., Francis, T. \& Kim, D. (2010). The Ideology of Homicide Detectives: A CrossNational Study. Homicide Studies, 14, 436-452.

Regoeczi, W., Jarvis, J. \& Riedel, M. (2009). Clearing Murders: Is it about Time? Journal of Research in Crime and Delinquency, 45, 142-162.

Regoeczi, W., Kennedy, L. \& Silverman, R. (2000). Uncleared Homicide: A Canada/United States Comparison. Homicide Studies, 4, 135-161.

Riedel, M. \& Boulahanis, J. (2007). Homicides Exceptionally Cleared and Cleared by Arrest: An Exploratory Study of Police/Prosecutor Outcomes. Homicide Studies, 11, 151-164.

Riedel, M., \& Jarvis, J. (1998). The Decline of Arrest Clearances for criminal Homicide: Causes, Correlates, and Third Parties. Criminal Justice Policy Review, 9, 279-306.

Riedel, M. \& Rinehart, T. (1996). Murder Clearances and Missing Data. Journal of Crime and Justice, 19, 83-102.

Roberts, A. (2008). Explaining Differences in Homicide Clearance Rates between Japan and the United States. Homicide Studies, 12, 136-145.

Servicio Médico Forense del Distrito Federal. (2010). Base de datos de muertes violentas registradas en el Distrito Federal

Servicio Médico Forense del Distrito Federal. (2009). Base de datos de muertes violentas registradas en el Distrito Federal.

Servicio Médico Forense del Distrito Federal. (2008). Tabulado de datos de muertes violentas registradas en el Distrito Federal. (2000-2008). 
Trussler, T. (2010). Explaining the Changing Nature of Homicide Clearance in Canada. International Criminal Justice Review, 20, 366-383.

UNODC. (2013). Global Study on Homicide. Recuperado de http://www.unodc.org/documents/ gsh/pdfs/2014_GLOBAL_HOMICIDE_BOOK_web.pdf

$\mathrm{Xu}$, Y. (2008). Characteristics of Homicide Events and the Decline in Homicide Clearance: A Longitudinal Approach to the Dynamic Relationship, Chicago 1966-1995. Criminal Justice Review, 33, 453-479.

Wellford, C. \& Cronin, J. (1999). An Analysis of Variables Affecting the Clearance of Homicides: A Multistate Study. Washington: Justice Research and Statistics Association. Recuperado de http://tinyurl.com/bb9s9gn

Zepeda, G. (2003). La investigación de los delitos y la subversión de los principios del subsistema penal en México, USMEX 2003-04 Working Paper Series, Project on Reforming the Administration of Justice in Mexico, Center for U.S. Mexican Studies, La Jolla.

Recibido el 29 de julio de 2014. Aceptado el 16 de junio de 2015. 